\title{
La urdiembre en la enseñanza: expresiones articuladoras de contenido en la interacción profesor-estudiante ${ }^{1}$
}

\author{
Himmbler Olivares ${ }^{2}$ \\ ORCID: 0000-0001-9223-3351 \\ David Silva ${ }^{3}$ \\ ORCID: 0000-0003-2635-7713 \\ Paloma Opazo ${ }^{4}$ \\ ORCID: 0000-0001-5057-5513 \\ Tomás Arellano ${ }^{5}$ \\ ORCID: 0000-0001-6642-8761 \\ Carlos Cornejo $0^{5}$ \\ ORCID: 0000-0001-5426-0025
}

\section{Resumen}

Este estudio busca ser un aporte a la pedagogía general al indagar un tipo particular de intervenciones pedagógicas denominadas expresiones articuladoras, caracterizadas por introducir conexiones entre diferentes asuntos técnicos o temas asociados a una disciplina que emergen durante una clase. Se determinaron dos tipos de expresiones articuladoras: de carácter local o global. En particular, se realizó un análisis de videos de clases de matemáticas con dos jueces expertos, quienes llevaron a cabo la codificación de la frecuencia y tipo de las expresiones articuladoras. El material seleccionado correspondió a videos de clases de matemáticas de 138 profesores de segundo ciclo de Enseñanza Básica en Chile. Posteriormente, las expresiones articuladoras identificadas fueron correlacionadas con el desempeño docente global de los 138 profesores, de acuerdo al Sistema Nacional Chileno de Evaluación de Desempeño Docente. Los resultados muestran que las expresiones articuladoras, en particular las de carácter local, correlacionan de forma positiva, pero con un efecto reducido, con distintas dimensiones del desempeño docente, tales como la capacidad del profesor de dar estructura a la clase o la promoción de la interacción pedagógica. Las expresiones articuladoras corresponderían a un recurso pedagógico importante del docente para el aprendizaje, en tanto facilitan la enseñanza

\footnotetext{
1- Esta investigación fue financiada por el Programa Bicentenario de Ciencia y Tecnología CIE 01 CONICYT a través del Centro de Políticas y Prácticas Educativas (CEPPE) de la Pontificia Universidad Católica de Chile. Agradecemos al Centro de Medición (Mide UC) por la facilitación del material audiovisual y de los resultados obtenidos por los docentes en el Sistema de Evaluación del Desempeño Profesional Docente.

2- Universidad de Concepción, Concepción, Chile. Contacto: hiolivares@udec.cl.

3- École des Hautes Études en Sciences Sociales, París, Francia. Contacto: davidsilvalabra@gmail.com.

4-Vrije Universiteit Amsterdam, Amsterdam, Holanda. Contacto: p.opazoreyes@vu.nl.

5- Pontificia Universidad Católica de Chile, Santiago, Chile. Contactos: tarellanosalas@gmail.com; cca@uc.cl.
} 
del lenguaje técnico de cada disciplina. Lo anterior se logra al establecer relaciones con asuntos tratados durante la clase, pero también con contenidos enseñados previamente o incluso con futuros asuntos disciplinares.

\section{Palabras clave}

Expresiones articuladoras - Técnicas y estrategias de enseñanza - Desempeño docente.

\section{The weaving in teaching: content-articulating expressions in teacher-student interaction}

\section{Abstract}

This research seeks to be a contribution to general pedagogy by studying a particular type of pedagogical intervention called articulating expressions, which are characterized as connections between different technical topics or topics associated with a discipline that emerge during a class. Two types of articulating expressions were determined: local and global. We conducted a video analysis of math classes with two expert judges, who carried out the coding of the frequency and type of articulating expressions. The selected material corresponded to videos of math classes from 138 teachers of second cycle of Elementary Education in Chile. Following this, the identified articulating expressions were correlated to the overall teaching performance of the 138 teachers, according to the Chilean National System of Teacher Performance Evaluation. The results show that articulating expressions, particularly those of a local nature, correlate positively but with a reduced effect, to different dimensions of teaching performance, such as the teacher's ability to structure the class or promote pedagogical interaction. Articulating expressions would correspond to an important pedagogical resource for the teacher to enhance learning, while facilitating the teaching of the technical language of each discipline. The latter is achieved by establishing relationships with topics discussed during class, but also with content that was previously taught, and even with future content related to the discipline.

\section{Keywords}

Articulating expressions - Teaching techniques and strategies - Teaching performance.

\section{Introducción}

Las interacciones en el aula constituyen un importante ámbito dentro de la investigación educacional actual. Los métodos para su estudio han sido diversos, recurriendo a la etnografía (GREEN et al., 2015; KANANU KIRAMBA, 2019; MERCER, 2010), al análisis conversacional (SEEDHOUSE, 2015), al análisis del discurso (BRAVO, 
2017), a los estudios interpretativos (MARTIN et al., 2005) y al análisis interaccional (COBB et al., 1992), entre otros. Una de las múltiples líneas de investigación presentes en este campo se ha enfocado en la descripción de patrones recurrentes en el discurso pedagógico. Lemke (1997), por ejemplo, describe el modo mediante el cual los contenidos de física, química o biología son comunicados dentro del aula. Según el autor, dichos lenguajes especializados permiten la construcción de significados entre profesores y estudiantes (LEMKE 1997; SÁNCHEZ; ROSALES, 2005). Al mismo tiempo, Bravo (2017) explora las prácticas que conforman el discurso científico en clases de Educación Primaria, dentro de los cuales está, por un lado, la introducción de un léxico propio caracterizado por una terminología precisa (McCOMAS, 2013) y, por otro, la enseñanza de dos géneros discursivos relevantes: la explicación científica y la formulación de argumentos científicos. En el ámbito matemático sucede un proceso similar: Martin y otros autores (2005), por ejemplo, exponen las prácticas realizadas por el profesor para que los estudiantes aprendan las reglas del juego necesarias para construir argumentos válidos y pruebas formales, aspectos elementales de tal disciplina.

Ciertamente, los significados asociados a las disciplinas escolares son construidos durante la interacción entre docente y estudiantes (LEMKE, 1997; MERCER, 2001; SÁNCHEZ; ROSALES, 2005). Las teorías socioculturales incluso subrayan las tensiones y negociaciones que continuamente suceden dentro del aula (KANANU KIRAMBA, 2019). Esta investigación, en particular, tiene por objetivo aportar a la pedagogía general al enfocarse en interacciones guiadas por el profesor que conectan contenidos de la enseñanza. Este tipo de intervenciones realizadas por el docente contribuyen en el proceso de construcción de significado, razón por la cual serán denominadas expresiones articuladoras. La exposición de contenidos de enseñanza constantemente recurre a ellas con el fin de establecer relaciones significativas entre contenidos específicos. Si bien cada profesor genera distintas relaciones entre el contenido disciplinar presentado, el presente estudio busca explorar si la presencia de estas expresiones puede asociarse a un mejor desempeño docente global evaluado en el aula. Para analizar lo anterior, se estudian videos de clases de matemáticas, una vez que corresponden a una disciplina con alta frecuencia de asuntos técnicos. Dichas expresiones, sin embargo, también pueden encontrarse en otras disciplinas, al conformarse como elementos centrales del proceso de construcción de significados realizado por el docente.

\section{Estrategias del profesor en la interacción pedagógica}

El proceso de enseñanza, aunque conducido por el docente, ocurre de forma compartida entre este y los estudiantes. A lo largo de este proceso aparecen recursos lingüísticos específicos orientados a guiar el aprendizaje de los estudiantes. Las preguntas, retroalimentaciones y recapitulaciones son parte de estas prácticas, pero también lo son las repeticiones, las reformulaciones y exhortaciones (MERCER, 1997, 2001). Los recursos mencionados permiten, principalmente, relacionar contenidos pertinentes para la construcción del conocimiento en la enseñanza de unidades temáticas de una asignatura. Sumado a esto, el establecimiento de relaciones puede suceder entre contenidos enseñados en el transcurso de una clase, pero también entre conocimiento disciplinar visto 
previamente o en un tiempo posterior. La educación en el aula es una experiencia continua y acumulativa para los participantes y, tal sentido de continuidad es fundamental para la generación de conocimiento compartido (MERCER, 2010).

Los múltiples recursos descritos por Mercer (1997, 2001) pueden organizarse en cuatro grandes categorías: recursos para el involucramiento de los estudiantes, recursos utilizados para destacar determinado contenido, recursos para obtener información a partir de pistas y, finalmente, recursos para monitorear contenidos del conocimiento compartido (SÁNCHEZ; ROSALES, 2005). Se deduce, entonces, que la enseñanza es entendida como un proceso comunicativo donde los interlocutores colaboran en la construcción de entendimiento conjunto. El énfasis comunicativo implicó la realización de múltiples estudios a partir de aproximaciones discursivas. Sin embargo, la búsqueda por patrones o recursos generales provocó que el contenido específico de la disciplina fuera relegado a un segundo plano. Por este motivo, investigadores contemporáneos han tenido como propósito elaborar modelos capaces de incorporar la dimensión pedagógica y disciplinar. Dentro de ellos, el Enfoque Ontosemiótico de los conocimientos y competencias del profesor de matemáticas (GODINO; BATANERO; FONT, 2007; GODINO et al., 2017) utiliza principios basados en la teoría constructivista e interaccionista para describir una didáctica que considera objetos y procesos matemáticos. Dicho conocimiento didáctico-matemático ha sido articulado recientemente en torno a un modelo de competencias denominado CCDM (GODINO et al., 2016).

La inclusión de las relaciones entre las prácticas del profesor y el contenido disciplinar permiten evidenciar los progresos en los contenidos enseñados. Asimismo, las relaciones entre el contenido disciplinar contribuyen a formar un sentido de continuidad dentro del aula, lo que es clave para la construcción de significado por parte de los estudiantes. A través del contenido disciplinar específico se puede estudiar cómo el profesor presenta los contenidos a lo largo de una clase o unidad temática. El conocimiento presentado puede ser enseñado mediante múltiples estrategias, ya sea a través de secuencias de interactividad (COLL et al., 1992), estructuras de conocimiento práctico (HUANG; NORMANDIA; GREER, 2005) o patrones temáticos (LEMKE, 1997).

De acuerdo a Lemke (1997), los patrones temáticos se refieren al conjunto de conceptos de lenguaje técnico disciplinar, los cuales suelen ser repetidos por el profesor durante la clase o un conjunto de clases con la intención de crear conexiones entre dichos términos. Los patrones temáticos crean relaciones semánticas, tal como se muestra en el discurso científico donde un patrón temático pequeño es unido con otro, proceso denominado por Lemke (1997) como condensación temática. El autor describe relaciones semánticas habituales, como es el caso de aquella de tipo nominal, donde se enlazan cualidades, cantidades y tipos a un patrón temático central. Un proceso similar describe Twiner y otros autores (2014) cuando se refieren a trayectorias de construcción de significado. De esta manera, los patrones temáticos sirven para entender las estrategias de enseñanza llevadas a cabo por el profesor. Durante el transcurso de la clase, el docente puede presentar relaciones semánticas que cambian de acuerdo a la incorporación de nuevos patrones temáticos o el aumento en la complejidad de patrones previos.

El profesor utiliza ciertas estrategias para la enseñanza de contenidos específicos, que consisten en recursos lingüísticos específicos que introducen patrones temáticos durante 
la clase (LEMKE, 1997). Dentro de tales estrategias se encuentran, por un lado, las situadas en el diálogo y, por otro, las estrategias monológicas. En el primer caso, se encuentra la construcción conjunta del conocimiento a través de lo que Lemke (1997) denomina diálogo triádico. La unidad básica del diálogo en el aula posee una secuencia de preguntarespuesta-evaluación positiva, donde se sitúa la elaboración de preguntas, además de la selección, modificación y reconstrucción retroactiva de respuestas expresadas por los estudiantes. En el segundo caso, el profesor presenta la exposición lógica o argumental de los contenidos, la presentación de una secuencia narrativa o estrategias más cercanas a la elaboración de resúmenes (LEMKE, 1997). En los últimos años se han dado importantes contribuciones sobre las estrategias dialógicas utilizadas por los docentes. De acuerdo a Mercer y Littleton (2007), las preguntas de los profesores, ya sean abiertas o cerradas, pueden tener una amplia gama de funciones comunicativas.

Tanto los patrones temáticos como las estrategias mencionadas poseen importantes consecuencias en la investigación contemporánea sobre prácticas pedagógicas y discurso docente. En primer lugar, ambos conceptos permiten estudiar el sentido de continuidad de la clase propuesto por Mercer (2001), poniendo especial énfasis en los aspectos temáticos del contenido disciplinar. En segundo lugar, los planteamientos de Lemke (1997) consideran la forma en la cual los estudiantes desarrollan, progresivamente, su propio conocimiento de la disciplina. Esto, a partir del uso de los patrones temáticos dentro de la interacción social cotidiana que se produce en la sala de clases. Como consecuencia, se hace necesario estudiar las interacciones en curso, considerando las intervenciones del profesor, así como las intervenciones de los estudiantes. Al incorporar dichos elementos, se puede comprender cualitativamente de qué forma se facilita el desarrollo del conocimiento disciplinar en los estudiantes. Dentro de los ejemplos interesantes de este tipo de abordaje, se encuentran los trabajos de Iturra (2013) e Iturra y Rosales (2015), donde las interacciones guiadas por el profesor contribuyen al desarrollo de estrategias para la comprensión lectora.

El presente trabajo aborda las intervenciones pedagógicas llevadas a cabo por el profesor dentro de la interacción pedagógica. Para ello, se considera el lenguaje técnico de la disciplina, en este caso referido a la matemática, como un interesante caso de estudio para explorar el funcionamiento de las expresiones articuladoras, que serán descritas en secciones posteriores. La relevancia de las intervenciones mediadas por el docente radica en su rol preponderante al guiar los procesos de enseñanza-aprendizaje (MERCER; LITTLETON, 2007), como se ha señalado previamente. No obstante, esta investigación se distingue de otras similares, de tipo socioculturales o discursivas, al contar con una aproximación microgenética (ROSENTHAL, 2004), la que posibilita abordar el momento a momento del fenómeno inmerso en la interacción de aula, espacio en donde las intervenciones pedagógicas cobran sentido.

\section{Microgénesis de la construcción de significado}

Desde un punto de vista teórico, la construcción de significado se entiende como un proceso microgenético. Dicho proceso remite a la formación de una expresión, de una percepción o de un pensamiento que, gradualmente y en tiempo presente, da cuenta del significado en la totalidad de la experiencia de quien lo vive (ROSENTHAL, 2004; 
SANDER, 1930; WERNER, 1955). El carácter genético apunta a los momentos específicos de articulación, diferenciación y reintegración que son desplegados progresivamente en la experiencia inmediata (DIRIWÄCHTER, 2008; SANDER, 1930; VALSINER; VAN DEER VEER, 2000; WERNER, 1955). Tal despliegue, sin embargo, ocurre en momentos previos a la reflexión metacognitiva y verbal. En palabras de Rosenthal, la "[...] teoría de la microgénesis describe la emergencia de la experiencia inmediata (es decir, percepción, pensamiento, expresión, imaginación) como un desarrollo" (2004, p. 86). Lo anterior implica que los procesos de formación microgenética que emergen en la experiencia, son organizados a partir de la experiencia inmediatamente precedente. Durante la situación comunicativa, la microgénesis da cuenta de la formación conjunta de significados en la experiencia de los interactuantes.

Siguiendo la situación comunicativa mínima elaborada por Bühler (1961), la función expresiva del lenguaje apela a la comprensión por parte de otro y se orienta a la construcción de un objeto de conciencia común entre hablante y oyente (HÖRMANN, 1986). En este sentido, la microgénesis de la formación de significado ocurre de forma evidente cuando se considera todo el conjunto de claves lingüísticas y no lingüísticas implicadas en una expresión verbal (WERNER, 1955). En ciertos momentos de la comprensión, el gesto juega un rol preponderante donde se torna imposible distinguir entre el contenido semántico y aquello expresado verbalmente (NÚÑEZ, 2008). Cuando se consideran las intervenciones del profesor dentro del aula desde un punto de vista microgenético, se destacan momentos específicos donde el profesor contribuye en la formación de significado referido al conocimiento disciplinar (MACIEL; UCHOA BRANCO; VALSINER, 2004). Aquellas intervenciones acontecen en el flujo de las tematizaciones presentadas por el docente en la interacción con los estudiantes.

\section{Expansiones de sentido, asuntos y modos de presentación}

Es posible distinguir dos elementos en cada expresión lingüística, ya sea de lenguaje cotidiano o lenguaje técnico, que sucede en la interacción: el asunto y su modo de presentación (GURWITSCH, 1979; HUSSERL, 1949; JAMES, 1989). El asunto de una expresión corresponde al hecho o estado de cosas del mundo al cual la expresión se refiere. Sin embargo, aquel asunto o tema referido en el habla es expresado de un cierto modo, por lo que un asunto tiene un determinado modo de presentación. El modo corresponde a todo lo que está disponible en la experiencia de los participantes de la interacción, vale decir, gestos manuales, gestos faciales y movimientos corporales que acompañan la expresión lingüística.

Esta investigación profundiza en aquellas expresiones dichas por el profesor relacionadas con asuntos matemáticos. Cada expresión es considerada una totalidad y, por ello, recibe el nombre de expansión de sentido. La denominación apunta a su cualidad de extender microgenéticamente el significado de un asunto matemático dentro de la situación comunicativa. El siguiente ejemplo tiene por objetivo ver la aplicación de los conceptos de asunto, modo de presentación y expansión de sentido: si un profesor quiere mostrar el asunto fracciones, puede hacerlo al entregar una definición formal 
de fracciones, o bien, al usar un gesto que apunte -usualmente con el dedo índice extendido- a las fracciones que tiene escritas en la pizarra. Ambas acciones expanden el sentido compartido sobre el asunto fracciones y corresponden a dos diferentes modos de presentación del mismo asunto.

En el despliegue microgenético del discurso es posible encontrar expresiones que, en vez de hacer referencia a asuntos particulares, relacionan dos asuntos. Si seguimos con el ejemplo de las fracciones, el profesor puede, en un momento de la clase, señalar a sus estudiantes que existe una relación entre las fracciones y los números decimales. En dicho caso la expansión de sentido no presenta un asunto temático nuevo, puesto que conecta dos asuntos técnicos. En la misma línea, aquellas expresiones cuya intencionalidad pedagógica consiste en ligar asuntos técnicos, en vez de introducir un asunto nuevo, serán denominadas expresiones articuladoras.

\section{Expresiones articuladoras}

El profesor no sólo presenta asuntos técnicos nuevos en la situación pedagógica: hilvana asuntos y concatena aquellos ya presentados o por ser presentados. Las concatenaciones presentadas por el profesor en una o más clases construyen una trama o tejido temático. En esta trama, los distintos asuntos forman una totalidad orientada a generar un modo técnico de hablar sobre el mundo o aspectos de él. Todas aquellas expresiones que permiten concatenar asuntos y ser, de alguna forma, parte de la trama temática que los relaciona, serán llamadas expresiones articuladoras. Si bien Godino y otros autores utilizan otro marco conceptual, apuntan a un proceso similar cuando preguntan: “¿Cuáles son los significados del objeto matemático (por ejemplo, cuáles son los diferentes significados de la probabilidad)? ¿Cómo se articulan entre sí?" (2016, p. 278).

Las expresiones articuladoras no se restringen a elementos lingüísticos, pero la literatura destaca que suelen ser proferidas, gramaticalmente, usando la primera persona plural. Este aspecto formal es indicativo de su función de dirigir la conciencia compartida hacia un determinado asunto. Otro rasgo relevante de las expresiones articuladoras es su dimensión temporal, pues articulan en tiempo presente aspectos del lenguaje técnico que fueron presentados de forma previa o que serán presentados de forma posterior. En otras palabras, la expresión articuladora hace referencia retroactiva y proactiva a los asuntos en el tiempo presente (GURWITSCH, 1979).

\section{Tipos de expresiones articuladoras: articulación global y articulación local}

Se estudian dos tipos de articulaciones: articulación global y articulación local, cada una de ellas con dos variantes (técnica y experiencial). La articulación global es una intervención -o un conjunto de intervenciones- que realiza el profesor para vincular o establecer relaciones sustantivas de contenido disciplinar. En este sentido, es un tipo de intervención similar a las recapitulaciones descritas por Mercer (1997) o las relaciones entre patrones temáticos descrita por Lemke (1997). La principal característica de este tipo de articulación es que ofrece un sentido de totalidad temática. Es abarcadora, ya que 
su presentación articula un campo de significación amplio, capaz de acoger múltiples asuntos que se han presentado en la situación comunicativa. En cuanto a la dimensión temporal, puede articular tanto de forma retroactiva como proactiva. En concreto, la articulación global puede articular asuntos tratados en una clase anterior con aquellos por tratar en una nueva clase (dirección retroactiva), o bien, puede dar cuenta de asuntos que se tratarán en momentos siguientes de la misma clase (dirección proactiva).

La articulación local es un tipo de intervención que el profesor realiza para establecer relaciones sustantivas de contenido disciplinar. A diferencia de la articulación global, este tipo de articulación es más acotada en las relaciones establecidas entre contenido temático. De esta manera, son locales porque usualmente remiten a la articulación entre dos asuntos que se suceden temporalmente. Si bien la dimensión temporal también puede orientarse retroactiva o proactivamente, es más acotada en comparación con las articulaciones globales.

Tanto la articulación global como la articulación local pueden ser de dos tipos: técnica o experiencial. La distinción radica en cuán explícitas son las relaciones entre los asuntos presentados. Si bien desde un punto de vista pedagógico es deseable que las relaciones de contenido temático se hagan de la forma más explícita posible, como es el caso de la articulación técnica, es posible observar que algunas expresiones articulatorias presentan dichas relaciones entre asuntos de un modo sugerido o tácito. Las expresiones que atisban una articulación entre asuntos, pero tal relación no es explícita, son denominadas experienciales. La dificultad de tales expresiones radica en que la experiencia inmediata de cada estudiante puede articularse en una dirección distinta a la intencionada por el profesor. Si la articulación no es explícita, su elaboración o cierre depende de la experiencia de los estudiantes. Al contrario, es posible que la expresión presentada por el profesor sea lo suficientemente explícita para que la relación entre los asuntos sea evidente. En tal caso, los docentes se aseguran que el proceso de construcción de significado de asuntos del lenguaje técnico esté adecuadamente articulado. Este tipo de articulaciones se llama articulaciones técnicas, en tanto tornan evidente las relaciones entre asuntos en el lenguaje técnico de la disciplina.

\section{El sistema de evaluación docente en Chile}

El Sistema de Evaluación del Desempeño Profesional Docente en Chile comenzó a implementarse el año 2003, como una respuesta a la necesidad de determinar de forma objetiva el desempeño de los profesores que trabajan en las escuelas públicas de Chile (SUN et al., 2011). La evaluación es implementada por universidades chilenas y, en ella, participan equipos formados tanto por académicos universitarios como por profesores de escuela capacitados en evaluación de material pedagógico. El sistema contempla distintos componentes: una pauta de autoevaluación, un informe emitido por superiores del establecimiento, una entrevista con un par (otro profesor), y un portafolio; este último recoge evidencias sobre la descripción de una unidad pedagógica, su evaluación y la filmación de una clase (FLOTTS; ABARZÚA, 2011). Específicamente, el presente estudio hizo uso de los resultados de la evaluación de la clase filmada, ya que en esta instancia 
de evaluación docente puede estudiarse la clase y, por extensión, las intervenciones que realizan los profesores.

La evaluación de la clase filmada considera las siguientes dimensiones: (a) estructura de la clase, que evalúa la capacidad de plantear actividades concordantes con los objetivos y el tiempo disponible, al mismo tiempo que evalúa si el profesor promueve que los estudiantes tengan un primer acercamiento a los contenidos; (b) calidad de la interacción pedagógica observada, que evalúa si las intervenciones que el profesor hace a sus estudiantes contribuyen al aprendizaje, además de buscar determinar si la implementación de las actividades de aprendizaje es acorde a los contenidos y metodologías de su asignatura; (c) ambiente de la clase, que evalúa la capacidad del profesor para focalizar a los estudiantes en las actividades propuestas, además de la generación de instancias equitativas de participación (FLOTTS; ABARZÚA, 2011).

Los objetivos de la presente investigación son, en primer lugar, determinar la frecuencia de aparición de las articulaciones globales y locales, tanto experienciales como técnicas, en clases de matemáticas de segundo ciclo básico de escuelas chilenas (de $5^{\circ}$ a $8^{\circ}$ Año Básico). En segundo lugar, nos interesa explorar si existe relación entre la frecuencia de aparición de las articulaciones y alguna de las dimensiones del desempeño docente, de acuerdo a los lineamientos del Sistema de Evaluación del Desempeño Profesional Docente.

\section{Metodología}

\section{Tipo de estudio}

La investigación realizó una observación sistemática, dado que se utilizaron categorías definidas a partir de investigaciones previas sobre aspectos del habla, con el fin de determinar relaciones cuantitativas sobre algunos de los aspectos de las clases (MERCER, 2010). La selección de la muestra fue probabilística.

\section{Participantes}

De un total de 1721 profesores voluntariamente evaluados en el año 2004 por el Sistema de Evaluación del Desempeño Profesional Docente de Chile, 249 corresponden al área de matemáticas y a segundo ciclo básico (esto es, entre $5^{\circ}$ y $8^{\circ}$ grado). De los 249 profesores de matemáticas filmados, se seleccionaron aleatoriamente 138. Los participantes pertenecen a establecimientos ubicados en distintas zonas administrativas y geográficas del país. De ellos, 103 (74,6\%) pertenecen a establecimientos ubicados en zonas urbanas, mientras que $35(25,4 \%)$ a zonas rurales. De los videos de la muestra utilizada para este estudio, 26 (18,8\%) fueron evaluados con desempeño destacado, 79 (57,2\%) con desempeño competente, 29 (21\%) con desempeño básico y 4 (2,9\%) fueron evaluados con desempeño insatisfactorio. La evaluación de su desempeño fue realizada por personas capacitadas en el sistema de evaluación que no pertenecen al equipo de investigación de este estudio.

Todos los profesores de matemáticas que participaron en la evaluación docente autorizaron mediante consentimiento informado el uso de sus datos para fines de investigación. Este consentimiento es genérico y corresponde a una autorización adicional 
a los datos de la evaluación docente y su aceptación (o rechazo) no tiene consecuencia alguna sobre la evaluación del docente. Cabe señalar que la revisión de los videos por parte de los codificadores de las expresiones articuladoras fue ciega, es decir, ningún miembro del equipo de investigadores conocía el resultado de la Evaluación de Desempeño Docente de cada profesor.

\section{Material}

Las videograbaciones son clases de 40 minutos de duración, filmadas por camarógrafos contratados por el Ministerio de Educación de Chile para la Evaluación de Desempeño Docente. Los evaluadores del Ministerio coordinaron y acordaron la filmación de la clase a través del director de escuela. Las instrucciones del registro se entregaron por escrito a cada profesor. Los profesores comunicaron al camarógrafo con anticipación las actividades a realizar para lograr un registro adecuado para su posterior revisión. Si bien se permitió que los profesores planificaran las actividades que ellos consideraban pertinentes, se les solicitó expresamente realizar actividades para promover la interacción entre el profesor y los estudiantes. Actividades tales como ver documentales o lectura individual en silencio no estaban autorizadas. Además, se indicó a los profesores incorporar un mínimo de 10 minutos de exposición durante la clase. Ningún miembro del equipo de investigación de este estudio participó del proceso de registro de los videos, ni tampoco hubo participación en otra actividad del proceso de evaluación docente.

\section{Análisis}

Dos jueces expertos fueron capacitados para realizar el proceso de identificación y categorización de las expresiones articuladoras en los 10 primeros minutos de cada clase. El criterio para emplear este lapso de la clase obedece a la naturaleza exploratoria de este estudio. Asimismo, este lapso de tiempo ha demostrado ser efectivo en investigaciones previas donde se estudia el desempeño docente a partir de la observación de periodos breves de una clase (AMBADY; ROSENTHAL, 1993; BABAD; AVNI-BABAD; ROSENTHAL, 2004). En esta misma línea, existe evidencia que muestra que en los 10 primeros minutos de la clase se tematizan aspectos de contenido claves para el desarrollo temático posterior (LEMKE, 1997). Se transcribieron las expresiones verbales enunciadas por el profesor y los estudiantes de cada clase, además de indicar el asunto matemático que trata la interacción en ese momento. Se registró además la gesticulación del profesor y su uso de materiales. Con toda esta información, los jueces codificaron el tipo de articulación, considerando los subtipos experiencial o técnico, en cada momento de la transcripción en la que se presentó el fenómeno.

El proceso de codificación involucró un periodo de prueba para acordar criterios con los jueces y calibrar las categorías. Una vez que se lograron niveles de confiabilidad aceptables $(\mathrm{k}=.8)$ entre los codificadores para cada tipo de articulación (local o global) y sus variantes experiencial y técnica, pudieron codificar cada video de clases de forma independiente. 
Cuando se finalizó el proceso de codificación, se determinaron las frecuencias de aparición de expresiones articuladoras y se estableció la relación entre las categorías y el puntaje de evaluación del video de la evaluación nacional docente. Los siguientes resultados del Sistema de Evaluación del Desempeño Profesional Docente fueron considerados para el análisis: el puntaje total de la clase filmada; el puntaje de la clase en la dimensión de interacción pedagógica; el puntaje de la clase en la dimensión de estructura de la clase; $\mathrm{y}$, finalmente, el puntaje de la clase en la dimensión de ambiente de la clase. Para el análisis se utilizó el coeficiente de correlación Rho de Spearman y la prueba de diferencia de medias de Mann-Whitney, debido a que las distribuciones muestrales de todas las variables estudiadas no obedecen a una distribución normal.

\section{Resultados}

Los resultados descriptivos para cada tipo de articulación y sus totales se muestran en la Tabla 1.

Tabla 1. Resultados descriptivos de articulaciones

\begin{tabular}{|c|c|c|c|c|c|c|c|c|c|c|}
\hline & & \multicolumn{6}{|c|}{ Explicitación } & & & \\
\hline & & \multicolumn{3}{|c|}{ Experiencial } & \multicolumn{3}{|c|}{ Técnica } & \multicolumn{3}{|c|}{ Total } \\
\hline & & $\mathrm{F}$ & $x$ & DS & $F$ & $x$ & DS & $\mathrm{F}$ & $x$ & DS \\
\hline \multirow{3}{*}{ Articulaciones } & Global & 15 & 0,11 & 0,312 & 4 & 0,03 & 0,168 & 19 & 0,14 & 0,346 \\
\hline & Local & 34 & 0,25 & 0,449 & 11 & 0,08 & 0,272 & 45 & 0,33 & 0,641 \\
\hline & $N=138$ & & & & & & & & & \\
\hline
\end{tabular}

Fuente: Elaboración propia.

Como puede apreciarse, las articulaciones de tipo global experiencial aparecen en 15 ocasiones, con una media de 0,11 en los 10 primeros minutos de la clase $(D E=0,312)$. La articulación global técnica se da en cuatro ocasiones, con una media de 0,03 por video $(D E=0,168)$. La articulación local experiencial es la que aparece con mayor frecuencia en las clases analizadas, con un total de 34 ocasiones, registrando una media de 0,25 por video $(D E=0,449)$. Lo anterior implica que, en la mayoría de los casos, los docentes de la muestra recurrieron a relaciones tácitas o sugeridas entre asuntos enseñados durante la clase. La articulación local técnica se presenta once veces, con una media de 0,8 por video $(D E=0,273)$. Considerando los valores totales, las articulaciones globales aparecen un total de diecinueve veces, con una media de 0,14 en los diez primeros minutos de las clases $(D S=0,346)$. Las articulaciones locales se encuentran un total de 45 ocasiones, con una media de 0,33 por cada video $(D E=0,529)$. En total, las articulaciones se presentan en 64 ocasiones, con una media de 0,46 por video $(D E=0,641)$.

Los ejemplos siguientes describen cualitativamente algunos de los resultados del estudio. En primer lugar, se muestra e interpreta un ejemplo de articulación global técnica (Cuadro 1) y, seguidamente, se presenta un ejemplo de articulación local experiencial (Cuadro 2). 
Cuadro 1- Ejemplo de Articulación global técnica

\begin{tabular}{|c|c|c|c|}
\hline Verbalización & Gesticulación & Uso de Materiales & Asunto \\
\hline $\begin{array}{l}\text { Profesor: Vamos a comenzar recordando las } \\
\text { fracciones. A ver ¿Quién puede decir qué es una } \\
\text { fracción? ¿Qué es una fracción? }\end{array}$ & & & \multirow{5}{*}{ NOCIÓN DE FRACCIÓN } \\
\hline Estudiante: Una división & & & \\
\hline $\begin{array}{l}\text { Profesor: Una división ¿Quién más? ¿Quién? ¿Una } \\
\text { fracción? }\end{array}$ & & & \\
\hline Estudiante: Una división exacta. & & & \\
\hline Profesor: Una división exacta. & & & \\
\hline $\begin{array}{l}\text { Muy bien. A ver vamos a ver las partes de la división. } \\
\text { ¿Cómo se llama este número? }\end{array}$ & Escribe en la pizarra una fracción & $\begin{array}{l}\text { Escribe la fracción " } 1 / 4 \text { " en } \\
\text { la pizarra }\end{array}$ & \multirow{2}{*}{$\begin{array}{l}\text { NUMERADOR DE LA } \\
\text { FRACCIÓN }\end{array}$} \\
\hline Estudiantes: Numerador & & & \\
\hline Profesor: ¿Qué indica el numerador? & & & \multirow{5}{*}{$\begin{array}{l}\text { NUMERADOR DE LA } \\
\text { FRACCIÓN }\end{array}$} \\
\hline Estudiantes: ... & & & \\
\hline Profesor: ¿Qué me indica? & & & \\
\hline Estudiante: Las partes que se ocupan & & & \\
\hline Profesor: Muy bien. & & & \\
\hline ¿Y este numerito que está debajito cómo se llama? & \multirow{2}{*}{$\begin{array}{l}\text { Con el dedo índice encierra en un } \\
\text { círculo al denominador de la fracción }\end{array}$} & \multirow{2}{*}{$\begin{array}{l}\text { Fracción escrita en pizarra } \\
\text { "1/4" }\end{array}$} & \multirow{2}{*}{$\begin{array}{l}\text { DENOMINADOR DE LA } \\
\text { FRACCIÓN }\end{array}$} \\
\hline Estudiantes Denominador & & & \\
\hline \multicolumn{4}{|l|}{ Profesor: y ¿qué indica? } \\
\hline Estudiantes: El entero. & & & \multirow{4}{*}{$\begin{array}{l}\text { DENOMINADOR DE LA } \\
\text { FRACCIÓN }\end{array}$} \\
\hline Profesor: La parte que divide al... & & & \\
\hline Estudiantes: Entero & & & \\
\hline \multicolumn{3}{|l|}{ Profesor: Muy bien. } & \\
\hline $\begin{array}{l}\text { Bueno. Ya sabíamos, ustedes me decían qué era una } \\
\text { fracción, qué era una división ¿verdad? }\end{array}$ & & & \multirow[t]{2}{*}{ NOCIÓN DE FRACCIÓN } \\
\hline Estudiantes: Sí & & & \\
\hline $\begin{array}{l}\text { Profesor: Voy a hacer un queque aquí, tengo este } \\
\text { queque y lo quiero dividir para ocho personas }\end{array}$ & \multirow{3}{*}{$\begin{array}{l}\text { Dibuja un círculo, y luego traza } \\
\text { líneas para dividirlo }\end{array}$} & \multirow{3}{*}{$\begin{array}{l}\text { Dibuja un círculo en la pizarra. } \\
\text { Hace una división imperfecta } \\
\text { del círculo }\end{array}$} & \multirow{8}{*}{ DIVISIÓN INEXACTA } \\
\hline ¿Cómo está ese queque en la repartición? ¿está bien? & & & \\
\hline Estudiantes: Sí & & & \\
\hline $\begin{array}{l}\text { Profesor: Está bien ¿0 sea que este niñito va a comer } \\
\text { lo mismo que este otro niñito? }\end{array}$ & \multirow{5}{*}{$\begin{array}{l}\text { Señala un pedazo del círculo y } \\
\text { luego otro pedazo }\end{array}$} & \multirow{5}{*}{$\begin{array}{l}\text { Círculo con divisiones } \\
\text { imperfectas dibujado en la } \\
\text { pizarra }\end{array}$} & \\
\hline Estudiantes: ¡No! & & & \\
\hline Profesor: No, no está... & & & \\
\hline Estudiantes: ....Bien & & & \\
\hline Profesor: No está exacto, ¿verdad? & & & \\
\hline $\begin{array}{l}\text { Pero si hago y hago esto con el queque....Lo divido de } \\
\text { esta manera ¿Cómo está ahora esta repartición? }\end{array}$ & \multirow{3}{*}{$\begin{array}{l}\text { Dibuja otro círculo en la pizarra y } \\
\text { luego traza líneas para dividirlo }\end{array}$} & \multirow{3}{*}{$\begin{array}{l}\text { Hace un círculo en la pizarra. } \\
\text { Después lo divide en partes } \\
\text { iguales }\end{array}$} & \multirow{3}{*}{ DIVISIÓN EXACTA } \\
\hline Estudiantes: ¡Bien! & & & \\
\hline Profesor: Bien & & & \\
\hline Entonces la fracción es una repartición... & \multirow{3}{*}{$\begin{array}{l}\text { Señala círculo con divisiones } \\
\text { iguales y con dedo índice repasa } \\
\text { algunas divisiones }\end{array}$} & & \multirow{3}{*}{ NOCIÓN DE FRACCIÓN } \\
\hline Estudiantes: Exacta & & & \\
\hline Profesor: Exacta, muy bien. & & & \\
\hline
\end{tabular}

Fuente: Elaboración propia. 
En el momento de la clase expuesto (duración: 1'34”), el profesor logra conectar explícitamente una serie de asuntos matemáticos presentados en la situación comunicativa de la clase. Por una parte, indica tanto verbal como gestualmente los componentes de la fracción (numerador y denominador), articulando la relación que ambos tienen en la fracción. Por otra parte, el profesor, en conjunto con los estudiantes, define la fracción como una división, articulando la relación entre estos dos asuntos. Para realizar esto, el profesor establece la distinción entre divisiones exactas e inexactas. Se trata entonces de una articulación global, por la cantidad y por la forma en la que se busca abarcar diversos asuntos. La relación planteada sería global de tipo técnico, debido a que los enlaces entre los asuntos son explícitos.

Lo cuadro 2 muestra el ejemplo de una clase donde aparece una articulación local experiencial.

Cuadro 2- Ejemplo de Articulación local experiencial

\begin{tabular}{|c|c|c|c|}
\hline Verbalización & Gesticulación & $\begin{array}{l}\text { Uso de } \\
\text { Materiales }\end{array}$ & Asunto \\
\hline $\begin{array}{l}\text { Profesor: Vamos a dar un problema bastante simple. Vamos con } \\
\text { las manzanas que es lo más normal y tenemos quince manzanas. } \\
\text { Tenemos las manzanas y regalamos. Regalamos siete. La pregunta } \\
\text { ¿Cuánto nos queda? A simple vista demasiado fácil, ¿cierto? }\end{array}$ & Escribe en la pizarra & & PROBLEMA MATEMÁTICO \\
\hline Estudiantes: Sí & & & \\
\hline $\begin{array}{l}\text { Profesor: Y vamos a variar. Vamos a cambiar y nos vamos a ir } \\
\text { a las ecuaciones ¿Qué vamos a hacer? Esta pregunta la vamos a } \\
\text { reemplazar por letras y... } \\
\text {...esa letra puede ser la equis, puede ser la y o la... }\end{array}$ & Escribe en la pizarra " $X, Y, Z$ " & & $\begin{array}{l}\text { EXPRESIÓN ALGEBRAICA DE } \\
\text { UNA ECUACIÓN PARA UN } \\
\text { PROBLEMA MATEMÁTICO }\end{array}$ \\
\hline Estudiantes: zeta & & & \\
\hline $\begin{array}{l}\text { Profesor: Y con eso ya tenemos uno que se llama incógnita que es } \\
\text { hacia dónde vamos nosotros a buscar (.) }\end{array}$ & & & $\begin{array}{l}\text { INCÓGNITA DE UNA } \\
\text { ECUACIÓN }\end{array}$ \\
\hline $\begin{array}{l}\text { ¿Qué vamos a hacer? Vamos a tomar las siete manzanas y ¿Qué pasó } \\
\text { ahora? ¿Tenemos cuánto es o no? }\end{array}$ & \multirow{9}{*}{ Escribe $7+x=15$} & & \multirow{9}{*}{$\begin{array}{l}\text { EXPRESIÓN ALGEBRAICA } \\
\text { Y ECUACIÓN PARA UN } \\
\text { PROBLEMA MATEMÁTICO }\end{array}$} \\
\hline Estudiantes: No & & & \\
\hline Profesor: ¿Qué vamos a poner? & & & \\
\hline Estudiantes: $x$ & & & \\
\hline Profesor: ¿Qué letra quieren? & & & \\
\hline Estudiante: $\mathrm{x}$ & & & \\
\hline Profesor: Ya ¿cuántas en total? & & & \\
\hline Estudiantes: Quince. & & & \\
\hline $\begin{array}{l}\text { Profesor: Ya. Tenemos las quince. Así vamos a ir transformando } \\
\text { en ecuaciones un problema tan simple como esas manzanas que } \\
\text { estamos viendo. }\end{array}$ & & & \\
\hline
\end{tabular}

Fuente: Elaboración propia. 
En esta situación comunicativa (duración: 1'45") el profesor intenta concatenar la noción de ecuación con un problema matemático sencillo que requiere usar la resta para saber el valor de la incógnita. La presentación de dos asuntos da cuenta que es una articulación local. El movimiento de la articulación comienza de forma muy explícita ("Esta pregunta la vamos a reemplazar por letras"), pero al escribir las expresiones $x, y$, $Z$ no verbaliza la traducción de cada una de estas expresiones y los valores del problema que se están intentando traducir. A partir de lo anterior, se puede concluir que es una articulación de tipo experiencial.

\section{Relación entre las articulaciones y el desempeño docente}

Las expresiones articuladoras están relacionadas con el desempeño docente de forma diferenciada: en primer lugar, se determinaron si las articulaciones eran globales o locales, para luego considerar las dimensiones particulares de desempeño estudiadas. Se utilizó para el análisis la prueba no paramétrica Rho de Spearman, la que puede obtener un valor que va entre el -1 y 1 , dependiendo si corresponde a una correlación perfecta negativa o positiva. El coeficiente de correlación es una medida estandarizada de un efecto observado, razón por la cual el valor indica el tamaño del efecto. De acuerdo a Field (2009), en general los valores \pm .1 representan un efecto pequeño, \pm .3 representan un efecto mediano $\mathrm{y} \pm .5$ representan un efecto importante, aunque varían dentro de cada área de investigación. La siguiente tabla presenta los valores de correlación entre las articulaciones y las dimensiones del Sistema de Evaluación del Desempeño Profesional Docente.

Tabla 2- Correlaciones entre las articulaciones y las dimensiones evaluadas en el Sistema Nacional Chileno de Evaluación del Desempeño Docente

\begin{tabular}{|c|c|c|c|c|}
\hline Articulación & $\begin{array}{c}\text { Puntaje Total Clase } \\
\text { Filmada }\end{array}$ & $\begin{array}{c}\text { Puntaje Estructura de } \\
\text { la Clase }\end{array}$ & $\begin{array}{l}\text { Puntaje Interacción } \\
\text { Pedagógica }\end{array}$ & $\begin{array}{c}\text { Puntaje Ambiente de la } \\
\text { Clase }\end{array}$ \\
\hline Global & 0,013 & 0,003 & 0,05 & $-0,018$ \\
\hline Local & $0,210^{\star *}$ & $0,221^{* *}$ & $0,200^{* *}$ & $0,192^{*}$ \\
\hline Total & $0,176^{*}$ & $0,187^{*}$ & $0,189^{*}$ & 0,131 \\
\hline
\end{tabular}

** La correlación es significativa al nivel 0,01.

* La correlación es significativa al nivel 0,05.

Fuente: Elaboración propia.

En términos generales, los resultados muestran una correlación débil entre el número total de expresiones articuladoras y el desempeño del profesor en la evaluación de la clase filmada en el Sistema de Evaluación de Desempeño Docente $\left(r_{s}=.176, p=<.05\right)$. Específicamente, el número total de articulaciones correlaciona de forma positiva, aunque débil, con las siguientes dimensiones: estructura de la clase $\left(r_{s}=.187, p=<.05\right)$ e interacción pedagógica $\left(r_{s}=.189, p=<.05\right)$. Las correlaciones mencionadas son estadísticamente 
significativas. En el caso del número total de articulaciones y el puntaje obtenido en ambiente de la clase, existe una correlación positiva, pero es débil y no es estadísticamente significativa.

El total de las articulaciones locales correlaciona positivamente con todas las dimensiones de la evaluación docente asociadas al desempeño en el aula, vale decir, con el puntaje total de la clase filmada $\left(r_{s}=.210, p=<.01\right)$, el puntaje de estructura de la clase $\left(r_{s}=.221, p=<.01\right)$, el puntaje de interacción pedagógica $\left(r_{s}=.200, p=<.01\right)$ y el puntaje de ambiente de la clase $\left(r_{s}=.192, p=<.05\right)$. Sin embargo, los valores del coeficiente denotan un efecto reducido. En cuanto a las articulaciones globales, los resultados no muestran correlaciones estadísticamente significativas con el desempeño docente en ninguna de las dimensiones estudiadas. Lo anterior puede obedecer a la baja frecuencia de aparición de las articulaciones globales en comparación con las articulaciones locales.

Los datos fueron analizados de acuerdo a la prueba Mann-Whitney, usada para comparar diferencias entre dos grupos independientes. Así, al evaluar la existencia de diferencias estadísticamente significativas en el grupo de profesores que utiliza expresiones articuladoras, los resultados muestran que el puntaje total de evaluación de la clase filmada es significativamente mayor en los profesores que presentan articulaciones, en comparación con los profesores que no las exhiben $(U=1854, p=.031)$. También se observa que los puntajes son significativamente mayores en el grupo de profesores que utiliza expresiones articuladoras en las dimensiones estructura de la clase $(U=1805, p=.017)$ e interacción pedagógica ( $U=1816, \mathrm{p}=.019$ ). No se observan diferencias significativas en los puntajes de la dimensión ambiente de la clase entre ambos grupos ( $U=2040, p=>.05$ ).

En cuanto al tipo de articulación, los resultados muestran diferentes correlaciones con las determinadas dimensiones de desempeño docente. Los valores se indican en la siguiente tabla.

Tabla 3- Correlaciones entre tipos de articulaciones y las dimensiones de desempeño docente evaluadas en el Sistema Nacional de Evaluación Docente

\begin{tabular}{|c|c|c|c|c|c|}
\hline \multicolumn{2}{|c|}{ Tipo Articulación } & Puntaje Total & Puntaje Estructura de & Puntaje Interacción & Puntaje Ambiente de la \\
\hline \multirow{2}{*}{ Global } & Experiencial & $-0,001$ & 0,011 & 0,022 & $-0,020$ \\
\hline & Técnica & 0,029 & $-0,014$ & 0,061 & 0 \\
\hline \multirow{2}{*}{ Local } & Experiencial & $0,162^{*}$ & $0,169^{*}$ & $0,160^{*}$ & $0,168^{*}$ \\
\hline & Técnica & $0,191^{*}$ & $0,181^{*}$ & $0,173^{*}$ & $0,148^{*}$ \\
\hline
\end{tabular}

Fuente: Elaboración propia.

Si bien se observan las correlaciones entre los tipos específicos de articulación global y las dimensiones del desempeño docente, todos los valores son bajos. Las correlaciones entre los tipos específicos de articulación global y las dimensiones de desempeño docente no son significativas desde el punto de vista estadístico. En cuanto a las articulaciones locales, de tipo experiencial y técnica, correlacionan positivamente con el puntaje total de la clase filmada, aunque su efecto sea débil $\left(r_{s}=.162, p=<.05\right.$ para la articulación local 
experiencial y $r_{s}=.191, p=<.05$ para la articulación local técnica). Tal efecto se repite con respecto al puntaje del módulo de estructura de la clase $\left(r_{s}=.169, p=<.05\right.$ y $r_{s}=.181$, $p=<.05$, de forma correspondiente), el puntaje de interacción pedagógica $\left(r_{s}=.160, p\right.$ $=<.05 \mathrm{y} r_{s}=.173, p=<.05$, de forma correspondiente) y el puntaje de ambiente de la clase $\left(r_{s}=.168, p=<.05\right.$ y $r_{s}=.148, p=<.05$, de forma correspondiente). En conclusión, las correlaciones entre los distintos tipos de articulación local y las dimensiones de desempeño docente son significativas, pero débiles en todos los casos.

\section{Discusión}

El interés de la investigación educativa en torno a las intervenciones pedagógicas tiene una larga data. La evidencia recogida en nuestro estudio identificó la presencia de intervenciones que denominamos expresiones articuladoras en clases de matemáticas. Dichas expresiones, que pueden ser de tipo local o global, se caracterizan por establecer redes entre diferentes contenidos, una suerte de urdiembre de la enseñanza. Dentro de los resultados, se presentaron asociaciones puntuales entre las expresiones articuladoras, en particular aquellas de tipo local, y ciertas dimensiones del desempeño docente. No obstante, tales correlaciones fueron débiles, por lo que requieren ser tomadas con mesura. La forma en la que el profesor une los distintos contenidos se relaciona, entonces, parcialmente con el desempeño docente.

Se indicaron relaciones positivas por encima del azar, aunque de bajo efecto, entre el total de articulaciones presentes en una clase y en variables tales como el desempeño global del profesor dentro del aula, siguiendo los parámetros del Sistema de Evaluación del Desempeño Profesional Docente. Asimismo, tales correlaciones se repitieron al considerar variables como estructura de la clase e interacción pedagógica. En el caso de las articulaciones locales, presentan correlaciones positivas, aunque nuevamente de efecto débil, en todas las variables de desempeño estudiadas: puntaje total de clase filmada, puntajes de estructura de clase, interacción pedagógica y ambiente de clase. Si bien se identificaron articulaciones globales, su frecuencia observada es baja y no muestran ningún tipo de relación estadísticamente significativa con el desempeño docente. Finalmente, se dieron resultados estadísticamente significativos, al abordar las diferencias en los puntajes de desempeño docente de los profesores que realizaron algún tipo de articulación y los que no presentaron.

Las correlaciones descritas fueron débiles en su efecto, pero marcan un antecedente de interés para futuras investigaciones. Si se considera la relación presente entre el total de las articulaciones con la calidad de la interacción pedagógica, se percibe la relevancia de tales intervenciones al guiar el proceso de aprendizaje de los estudiantes. De acuerdo a Flotts y Abarzúa, interacción pedagógica evalúa “[...] la capacidad del docente de explicar contenidos o procedimientos usando estrategias que promuevan una comprensión acabada por parte de los alumnos" (2011, p. 58). En este sentido, futuras investigaciones podrían evaluar los efectos de tales intervenciones en los aprendizajes de los estudiantes. En la misma línea, la correlación señalada con la dimensión estructura de clase permite elaborar hipótesis sobre la posibilidad de que las expresiones articuladoras sean un apoyo 
a elementos pedagógicos como la distribución del tiempo y de las actividades, aspectos que contribuyen a dar continuidad a las interacciones en la clase (EDWARDS; MERCER, 1988; LEMKE, 1997; MERCER, 1997, 2001). La dimensión ambiente de clase evalúa si el docente logra que los estudiantes realicen satisfactoriamente las actividades, “[...] manteniendo normas de convivencia que favorezcan el desarrollo de la clase, entregando instrucciones claras y supervisando el desarrollo de las actividades" (FLOTTS; ABARZÚA, 2011, p. 58). Así, se presume que las expresiones articuladoras expresadas por el profesor podrían contribuir a que los estudiantes se focalicen en las actividades de la clase.

Los resultados muestran importantes diferencias en las frecuencias de las articulaciones: la articulación global aparece en menos oportunidades que la articulación local. En términos metodológicos, la baja frecuencia observada en las articulaciones globales no permitió establecer relaciones estadísticas significativas con las variables de desempeño. Es posible que la baja frecuencia de aparición de la articulación global se deba a cuestiones de tipo temporal. Las articulaciones globales abarcan mayor contenido temático y, por ende, toman más tiempo en su elaboración que las articulaciones locales. Las diferencias de frecuencia pueden deberse a que las articulaciones locales ocurren en momentos localizados y breves, lo que favorece su aparición en los diez primeros minutos de la clase. En esta línea, es posible que se requiera de una unidad de tiempo mayor para el registro y análisis de las articulaciones globales, vale decir, un tiempo superior a los minutos de aula que se emplearon para este estudio. Asimismo, puede ser que estas intervenciones se encuentren con mayor frecuencia hacia el final de la clase, momento en el cual el profesor realiza intervenciones orientadas a unir de forma global los temas tratados en el transcurso de la clase.

Desde un punto de vista teórico, es posible que la articulación global requiera de un conocimiento profundo de la disciplina por parte del profesor, producto de su carácter abarcador. Gracias a este conocimiento, el docente puede tener una visión panorámica de los distintos contenidos implicados en el desarrollo de lenguaje técnico. El conocimiento de la disciplina debe acompañarse, además, de una actitud particular en la experiencia del docente. Esta actitud se centra en una reflexión que permite visualizar los temas tratados en un tiempo presente de la clase y, así, establecer relaciones con temas precedentes y por venir. En otras palabras, la articulación global surge en el campo de conciencia del profesor como un acto reflexivo en el que hace referencia retroactiva y proactiva, con la intención de dar un hilo conductor a la clase. Esta actitud es distinta en el caso de las articulaciones locales, cuyo objetivo es básicamente hilvanar un tema con otro precedente o subsecuente. De esta manera, resulta de interés para futuras investigaciones indagar en la experiencia subjetiva del profesor durante la clase, para vislumbrar si tal presentación y articulación de los contenidos responde a una planificación previa o no.

En general, los resultados muestran que más de la mitad de las clases analizadas no evidencian expresiones articuladoras. El aspecto mencionado podría relacionarse con el hecho de que en los 10 primeros minutos de clase prevalece la presentación de asuntos, más que la conexión entre ellos. Asimismo, hay menor frecuencia de articulaciones de tipo técnico, es decir, aquellas conexiones explícitas entre asuntos, tanto en las expresiones de tipo global como local. Esto llama la atención, ya que son en estas articulaciones donde 
se explicitan aspectos de lenguaje técnico. Mediante tales intervenciones, se garantiza un paso entendible para los estudiantes desde de lo cotidiano a lo técnico. La poca presencia de articulaciones de tipo técnico podría ligarse, al igual que la baja frecuencia de articulaciones globales, al nivel de dominio de los conocimientos propios del lenguaje disciplinar. Futuras investigaciones podrían abordar si existe una relación entre las frecuencias de expresiones articuladoras y el conocimiento disciplinar del profesor.

Una de las limitaciones de esta investigación radica en el desconocimiento de los efectos directos de este tipo de intervenciones en el aprendizaje de los estudiantes. Twiner y otros autores (2014) dan cuenta de la importancia de la participación de los estudiantes en la conformación de trayectorias de significados presentes. De esta manera, resulta de interés efectuar estudios para determinar si existe una relación entre las expresiones articuladoras y el aprendizaje de los estudiantes en la clase (SÁNCHEZ; ROSALES, 2005). Para ello, sería fructífero estudiar variantes de construcción de significado específicas que se dan en los estudiantes y su relación con las intervenciones que realiza el profesor en momentos específicos y/o en el conjunto de una clase o lección, tal como se realiza en trabajos sobre comprensión lectora (ITURRA; ROSALES, 2015). El uso de los primeros 10 minutos de enseñanza puede considerarse como una limitante, en tanto no se pudo contar con una descripción más acabada de la presentación de contenidos ocurrida durante la totalidad de la clase. Al mismo tiempo, los bajos valores en las correlaciones indican que el porcentaje de varianza capaz de explicar la asociación de las variables estudiadas es inferior al 20\% (NORMAN; STREINER, 2008). Ambas limitaciones podrían estar asociadas, si se mantiene la suposición de que un aumento en el tiempo analizado de las clases podría incrementar la frecuencia observada de articulaciones, y con ello, incrementar los valores de correlación. Estas limitaciones, sin embargo, son propias de la naturaleza exploratoria de la investigación.

Los resultados de la presente investigación deben considerarse como un insumo para seguir enriqueciendo los estudios en el área. En el futuro, se podría considerar una unidad de tiempo mayor de cada clase, considerando, por ejemplo, todos los momentos de la clase en la que el docente realiza actividades monológicas de exposición (LEMKE, 1997). Asimismo, sería interesante considerar de qué forma se articula el lenguaje técnico durante una clase completa para lograr una mejor comprensión del fenómeno estudiado. Esfuerzos como los realizados por Martin y otros autores (2005), quienes estudiaron las prácticas de un profesor y sus estudiantes por un lapso de cuatro meses, sirven para describir en profundidad el desarrollo temático que surge de la interacción. Lo anterior sería complementario a los planteamientos de Lemke (1997), al estudiar los asuntos técnicos en términos de su desarrollo temático, incluyendo las estrategias usadas por el profesor en la interacción dentro del aula.

\section{Referencias}

AMBADY, Nalini; ROSENTHAL, Robert. Half a minute: predicting teaching evaluations from thin slices of nonverbal behavior and physical attractiveness. Journal of personality and social psychology, Washington, DC, v. 64, n. 3, p. 431-441, 1993. 
BABAD, Elisha; AVNI-BABAD, Dinah; ROSENTHAL, Robert. Predictions of students evaluations from brief instances of professors nonverbal behavior in defined instructional situations. Social psychology of education, Basel, v. 7, n. 1, p. 3-33, mar. 2004.

BRAVO, Marco. Demystifying the discourse of science for elementary grade English learners. In: LANGMAN, Juliet, HANSEN-THOMAS, Holly (Ed.). Discourse analytic perspectives on STEM education. Springer: Cham, 2017. p. 177-194.

BÜHLER, Karl. Teoría del lenguaje. Revista de Occidente: Madrid, 1961.

COBB, Paul et al. Characteristics of classroom mathematics traditions: an interactional analysis. American Educational Research Journal, London, v. 29, n. 3, p. 573-604, otoño 1992.

COLL, César et al. Actividad conjunta y habla: una aproximación al estudio de los mecanismos de influencia educativa. Infancia y Aprendizaje, Oxfordshire, v. 59-60, p. 189-232, 1992.

DIRIWÄCHTER, Rainer. Genetic ganzheitspsychologie. In: DIRIWÄCHTER, Rainer; VALSINER, Jaan (Ed.). Striving for the whole: creating theoretical syntheses. New Jersey: Transaction, 2008. p. 21-45.

EDWARDS, Derek; MERCER, Neil. Conocimiento compartido: el desarrollo de la comprensión en el aula. Barcelona: Paidós, 1988.

FIELD, Andy. Discovering statistics using SPSS. London: Sage, 2009.

FLOTTS, María Paulina; ABARZÚA, Andrea. El modelo de evaluación y sus instrumentos. In: MANZI, Jorge; GONZÁLEZ, Roberto; SUN, Yulan (Ed.). La evaluación docente en Chile. Santiago de Chile: UC, 2011. p. 35-60.

GODINO, Juan; BATANERO, Carmen; FONT, Vicenç. The onto-semiotic approach to research in mathematics education. ZDM, Berlin, v. 39, n. 1-2, p. 127-135, en. 2007.

GODINO, Juan et al. Articulando conocimientos y competencias del profesor de matemáticas: el modelo CCDM. In: MACÍAS, Juan Antonio et al. (Ed.). Investigación en educación matemática XX. Málaga: Seiem, 2016. p. 285-294.

GODINO, Juan et al. Enfoque ontosemiótico de los conocimientos y competencias del profesor de matemáticas. Boletim de Educação Matemática, Rio Claro, v. 31, n. 57, p. 90-113, abr. 2017.

GREEN, Judith et al. Developing a multi-faceted research process: an ethnographic perspective for reading across traditions. In: MARKEE, Numa (Ed.). The handbook of classroom discourse and interaction. Chichester: John Wiley \& Sons, 2015. p. 26-43.

GURWITSCH, Aaron. El campo de la conciencia: un análisis fenomenológico. Madrid: Alianza, 1979.

HÖRMANN, Hans. Meaning and context: an introduction to the psychology of language. New York: Plenum, 1986. 
HUANG, Jingzi; NORMANDIA, Bruce; GREER, Sandra. Communicating mathematically: comparison of knowledge structures in teacher and student discourse in a secondary math classroom. Comunication Education, Oxfordshire, v. 54, n. 1, p. 34-51, 2005.

HUSSERL, Edmund. Ideas relativas a una fenomenología pura y una filosofía fenomenológica. México, DC: Fondo de Cultura Económica, 1949.

ITURRA, Carolina. Los diálogos construidos y los contenidos elaborados en clases dedicadas a la comprensión de textos, en aulas chilenas. Psicología Educativa, Madrid, v. 19, n. 2, p. 113-122, dic. 2013.

ITURRA, Carolina; ROSALES, Javier. Reading together in the classroom: a study of the difference between teacher and student interactions and the requirements of the Ministry of Education in Chile / Lectura conjunta en el aula. Un estudio sobre la distancia entre la interacción profesores alumnos y las prescripciones del Ministerio de Educación Chileno. Cultura y Educación, Oxfordshire, v. 27, n. 1, p. 64-92, 2015.

JAMES, William. Principios de psicología. México, DC: Fondo de Cultura Económica, 1989.

KANANU KIRAMBA, Lydiah. Heteroglossic practices in a multilingual science classroom. International Journal of Bilingual Education and Bilingualism, London, v. 22, n. 4, p. 445-458, mayo 2019.

LEMKE, Jay. Aprender a hablar ciencia: lenguaje, aprendizaje y valores. Barcelona: Paidós, 1997.

MACIEL, Diva; UCHOA BRANCO, Angela; VALSINER, Jaan. Bidirectional process of knowledge construction in teacher-student interaction. In: UCHOA BRANCO, Angela; VALSINER, Jaan (Ed.). Communication and metacommunication in human development. New York: Information Age, 2004. p. 109-125.

MARTIN, Tami et al. The interplay of teacher and student actions in the teaching and learning of geometric proof. Educational Studies in Mathematics, Dordrecht, v. 60, n. 1, p. 95-124, sept. 2005.

McCOMAS, William. The language of science education: an expanded glossary of key terms and concepts in science teaching and learning. Rotterdam: Sense, 2013.

MERCER, Neil. La construcción guiada del conocimiento: el habla de profesores y alumnos. Barcelona: Paidós, 1997.

MERCER, Neil. Palabras y mentes: cómo usamos el lenguaje para pensar juntos. Barcelona: Paidós, 2001.

MERCER, Neil. The analysis of classroom talk: methods and methodologies. British Journal of Educational Psychology, New Jersey, v. 80, p. 1-14, dec. 2010.

MERCER, Neil; LITTLETON, Karen. Dialogue and the development of children's thinking: a sociocultural approach. London: Routledge, 2007.

NORMAN, Geoffrey; STREINER, David. Biostatistics: the bare essentials. Cary: PHMP, 2008.

NÚÑEZ, Rafael. A fresh look at the foundations of mathematics: gesture and the psychological reality of conceptual metaphor. In: CIENKI, Alan; MÜLLER, Cornelia (Ed.). Gesture and metaphor. Amsterdam: John Benjamins, 2008. p. 93-114. 
OSENTHAL, Victor. Formas, sentido y desarrollo: acerca de la microgénesis. Cuadernos de Filología Francesa, Barcelona, v. 16, p. 85-104, 2004.

SÁNCHEZ, Emilio; ROSALES, Javier. La práctica educativa: una revisión a partir del estudio de la interacción profesor-alumnos en el aula. Cultura y Educación, Oxfordshire, v. 17, n. 2, p. 147-174, 2005.

SANDER, Friedrich. Structure, totality of experience, and Gestalt. In: MURCHISON, Carl (Ed.). Psychologies of 1930. Worcester: Clark University Press, 1930. p. 188-201.

SEEDHOUSE, Paul. L2 classroom interaction as a complex adaptive system. In: MARKEE, Numa (Ed.). The handbook of classroom discourse and interaction. Chichester: John Wiley \& Sons, 2015. p. 373-389.

SUN, Yulan et al. La implementación de la evaluación docente. In: MANZI, Jorge; GONZÁLEZ, Roberto; SUN, Yulan (Ed.). La evaluación docente en Chile. Santiago de Chile: UC, 2011. p. 63-89.

TWINER, Alison et al. Meaning making as an interactional accomplishment: a temporal analysis of intentionality and improvisation in classroom dialogue. International Journal of Educational Research, Amsterdam, v. 63, p. 94-106, 2014.

VALSINER, Jaan; VAN DER VEER, René. The social mind: construction of the idea. New York: Cambridge University Press, 2000.

WERNER, Heinz. A psychological analysis of expressive language. In: WERNER, Heinz (Ed.). On expressive language. Worcester: Clark University Press, 1955. p. 11-18.

Recibido en: 04.12.2018

Revisado en: 08.05.2019

Aprobado en: 25.06.2019

Himmbler Olivares es profesor asistente del Departamento de Psicología de la Universidad de Concepción (Chile).

David Silva es candidato a Doctor en Ciencias Sociales de la École des hautes Études en Sciences Sociales (Francia).

Paloma Opazo es estudiante de doctorado de la Facultad de Humanidades de la Vrije Universiteit Amsterdam (Holanda).

Tomás Arellano es sociólogo de la Pontificia Universidad Católica de Chile.

Carlos Cornejo es profesor titular de la Escuela de Psicología de la Pontificia Universidad Católica de Chile. 